\title{
RAZONABILIDAD COMO EXIGENCIA PARA EL CONOCIMIENTO DE LA ANTIJURIDICIDAD: EL CASO DE LA PERSONA INIMPUTABLE
}

Reasonability as a Requirement for Knowledge of Unlawfulness: The Case of the Mentally Disorder Person

\author{
JOSÉ MANUEL FERNÁNDEZ RUIZ ${ }^{1}$ \\ Universidad Alberto Hurtado
}

\section{RESUMEN}

El presente trabajo tiene por objetivo formular una respuesta a la siguiente pregunta: ¿puede aplicarse una medida de seguridad cuando el sujeto activo del delito es inimputable pero actúa con error de tipo? Si se considera que el inimputable no satisface el requerimiento de dolo, entonces debería concluirse que no actúa "típicamente" y, si no actúa típicamente, no podría aplicársele una medida de seguridad. Se examina qué tipo de respuesta ofrecería a este caso la doctrina chilena concluyendo que sus argumentos no son suficientes para desarrollar una respuesta plausible. Para elaborar una respuesta a esta pregunta, se introducen algunos elementos básicos de la epistemología analítica contemporánea y con su utilización se desarrolla la exigencia de "razonabilidad" como la condición crucial para establecer que el inimputable podría ser objeto de una medida de seguridad, aún si obra con error de tipo. Se concluirá que esta exigencia también es aplicable, en casos especiales, al sujeto imputable sea que actúe con error de tipo o de prohibición.

\section{PALABRAS CLAVE}

Razonabilidad, conocimiento de la antijuridicidad, inimputabilidad, desorden mental

\section{ABSTRACT}

The purpose of this paper is to formulate an answer to the following question: can a person be the object of an order of criminal commitment when the person that commits the crime has a mental disorder yet makes a mistake of fact? If it is considered that the person with a mental disorder does not satisfy the requirements of mens rea, it should be concluded that he does not act unlawfully, and if the person does not act unlawfully there could be not criminal commitment. The paper examines what kind of response the Chilean doctrine would offer to this case, concluding that their arguments are not sufficient to develop a plausible response. To elaborate an answer to the question, the paper introduces some basic elements of contemporary analytical epistemology, and with them, it develops the requirement of "reasonableness" as the crucial condition to establish that mentally disordered persons can be the object of an order of criminal commitment even when they make a mistake of fact. It is concluded that this requirement is also applicable, in special cases, to the culpable person whether he/she makes a mistake of fact or a mistake of law.

\section{KEYWORDS}

Reasonability, Knowledge of Unlawfulness, Exculpation, Mental disorder

\section{Introducción}

La pregunta “¿puede proceder la aplicación de una medida de seguridad cuando la persona actúa con error de tipo?"2 representa un problema para el derecho penal chileno,

${ }^{1}$ PhD Glasgow University, LLM Toronto University. Profesor de Derecho Penal Universidad Alberto Hurtado e investigador Asociado VIODEMOS (ANID - Programa Iniciativa Científica Milenio - Instituto Milenio para la Investigación en Violencia y Democracia, VIODEMOS). Dirección postal: Cienfuegos 41, Santiago, Chile. Correo electrónico: jmfernandez@uahurtado.cl. Agradezco, como siempre, los valiosos comentarios, paciencia y el apoyo incondicional de mi esposa, Malva Olavarría.

2 Para la presente investigación se consideran exclusivamente aquellos casos en que la legislación penal exige, como condición para aplicar la medida de seguridad, que la conducta del sujeto haya sido típica (lo que requiere la concurrencia de elementos objetivos 
porque el art. 455 del Código Procesal Penal (en adelante CPP) requiere como condición para aplicar una medida de seguridad que el hecho sea típico y antijurídico ${ }^{3}$. Si una conducta típica requiere satisfacer tanto exigencias de imputación objetiva como subjetiva y esta última no se satisface por falta de dolo, entonces la doctrina debería concluir que no puede aplicarse una medida de seguridad, ¿pero es esta conclusión correcta? Esta investigación propone que la respuesta es, posiblemente no. Para desarrollar esta respuesta es necesario elaborar de mejor manera esta exigencia de dolo y más en general de conocimiento, porque en rigor es precisamente una exigencia de conocimiento la que el inimputable no puede satisfacer, y lo que sugiere que este no pueda ser considerado como candidato/a para una medida de seguridad.

Ahora bien, el desconocimiento de la antijuridicidad de la conducta puede obedecer a diversos motivos. En efecto, puede tratarse de un error de prohibición, pero también puede tratarse de un desconocimiento motivado por un desorden mental ${ }^{4}$. En este trabajo no se abordarán las complejidades que representa el error de prohibición, en el sentido de si podría o no proceder cuando el sujeto es inimputable, básicamente porque parecen tratarse de situaciones analíticamente diferentes. En el error de prohibición el sujeto no sabe, por diferentes razones, que lo que hace es antijurídico, pero de dichas razones debe descartarse el caso en que el origen del desconocimiento se explica en un desorden mental. Es decir, para apreciar si es que hay o no un error de prohibición la causa del desconocimiento del carácter antijurídico del comportamiento no puede ser un desorden mental. Si la causa del desconocimiento es un desorden mental, entonces se trata de una situación de inimputabilidad. En definitiva y sin pretender resolver esta problemática, en este trabajo se examinará fundamentalmente el error de tipo que, causado por un desorden mental, genera un desconocimiento del hecho típico.

No obstante, lo anterior y, para aclarar el problema que plantea la pregunta que guía esta investigación, también se examinarán ciertos casos especiales en que una persona imputable actúa con error de prohibición y error de tipo. Para la formulación de la respuesta a dicha pregunta el método empleado no será completamente "dogmático", si por dogmático se entiende aquel tipo de investigación cuyo objetivo es la reconstrucción racional del sistema de responsabilidad penal ${ }^{5}$. En efecto, no es el objetivo central de este trabajo proponer una "mejor reconstrucción" del sistema jurídico-penal, aunque ello pueda ser un efecto secundario. Si bien la pregunta de investigación es acotada, el propósito más general es indagar en las condiciones de responsabilidad a partir de nuestra práctica del derecho penal, con el objetivo de hacer explícitas algunas implicancias en nuestra disposición al castigo-tratamiento del inimputable, en el entendido que esto promete iluminar algunas partes oscuras de aquella práctica y que son importantes para nosotros/as como participantes en ella. Para desarrollar el argumento se adoptan algunos conceptos básicos de la epistemología analítica angloamericana para reformular ciertos conceptos empleados por la dogmática penal. Mediante su utilización se pretende introducir mayor precisión en algunos conceptos "dogmáticos", de modo de facilitar la explicitación de los problemas que se presentan en el tratamiento de los casos objeto de análisis y permitir la elaboración de una respuesta plausible a ellos.

Partiendo de esta premisa metodológica la investigación se desarrolla con la siguiente estructura: en la primera sección se aborda la pregunta acerca de como entender el término "conocimiento" en la evaluación de la inimputabilidad y para lo cual se introducen conceptos de la epistemología analítica. La segunda sección comienza empleando los conceptos elaborados previamente y los adapta para la comprensión de las conductas tanto dolosas como culpables. Luego, se examina el tratamiento que dos posiciones doctrinarias chilenas darían al error de tipo

y subjetivos) y que el sujeto sea inimputable. Por lo tanto, no se consideran aquellos casos de leyes especiales como la Ley 20.000 y 19.925, véase TAPIA (2013), pp. 583 y ss. y QUIROGA y ARÉVALO (2014), pp. 501-507.

${ }^{3}$ Esta es la posición estándar de la doctrina chilena, véase con referencias FALCONE (2007), p. 240 y TAPIA (2013), p. 577.

4 Sobre la razón de la referencia "desorden mental" por sobre "trastorno mental" o "enfermedad mental" véase FERNÁNDEZ (2021a), pp. 8-9.

${ }^{5}$ La idea de un "sistema" es la premisa básica en esta comprensión de la labor dogmática, véase ROXIN (1997), pp. 192-193; BASCUÑAN (1998), pp. 17-20; ALEXY (2007), pp. 361-362; FERRAJOLI (2013), pp. 28-29. 
en el caso que un sujeto tenga un desorden mental. Esta sección cierra proponiendo una exigencia clave para el requerimiento de conocimiento, la exigencia de "razonabilidad". En la tercera sección y final se desarrolla y aplica esta exigencia en paralelo, tanto al caso en que una persona inimputable actúa con error de tipo, como a ciertos casos en que una persona imputable actúa con error de tipo y de prohibición. El ensayo concluye afirmando que la exigencia de razonabilidad permite resolver las dificultades argumentativas que presenta la literatura chilena, pero también revela como esta exigencia no solo aparece a nivel de la culpabilidad, sino que ya está presente a nivel de dolo. Por último, en la conclusión, se sugiere que ciertos estados intencionales de carácter disposicional, posiblemente integren la práctica del derecho penal, tensionando con ello el principio de responsabilidad por el hecho.

\section{El significado del "conocimiento" de la antijuridicidad de la conducta}

Esta sección tiene por propósito elaborar el significado del término "conocimiento" en la proposición que tradicionalmente afirma la doctrina chilena al sostener que la culpabilidad requiere "la capacidad de conocer lo injusto y de determinarse según ese conocimiento..." 6 . Esta capacidad es de tipo cognitivo y hace referencia a las habilidades que normalmente tienen las personas de razonar prácticamente y, por ende, establecer planes para el futuro. En la filosofía práctica de la acción humana ${ }^{7}$ se entiende que una forma básica de racionalizar o explicar nuestras acciones es apelar a ciertos estados intencionales: las creencias y los deseos de las personas. La creencia, bajo este entendimiento, es una capacidad representacional ${ }^{8}$ que le permite a la persona orientarse en su medio-ambiente físico y social, mientras que los deseos se comprenden como estados intencionales que operan como las distintas finalidades que un agente persigue. Por ejemplo, si $X$ desea "tomarse un helado", cree que "tiene dinero en el bolsillo" y que "en la esquina al lado de su trabajo venden helados" (comenzando su trayecto a comprarlo), el deseo de tomarse el helado y la creencia que en la esquina venden helados son las condiciones básicas que deberíamos considerar para racionalizar o explicar el trayecto de $X$ hacia la esquina. En este caso si un amigo de $X$ pregunta, ¿porque $X$ se dirige hacia esa calle? Responderíamos: porque $\mathrm{X}$ desea comprar un helado y cree que en la esquina puede comprarlo.

Sobre la necesidad de considerar estos dos aspectos básicos de la racionalización de una acción humana no hay mayor controversia ${ }^{9}$ y este parece un buen punto de partida para examinar que significa "conocimiento" cuando hablamos de la capacidad de imputabilidad, es decir, entender conocimiento en términos de la posesión de ciertas creencias y deseos por parte de un individuo. Cabe notar que no todas las capacidades relevantes para establecer que una persona "conoce" son de tipo cognitivo, pero son estas las que serán el objeto de análisis en este trabajo. Queda de este modo descartada la capacidad volitiva, a saber, la "capacidad de determinarse conforme al conocimiento (que una conducta es antijurídica)". Precisado lo anterior, aparece que el foco de análisis reside solo en una parte de las condiciones con las cuales se racionaliza la conducta humana, a saber, los componentes epistémicos o relativos al conocimiento. Ahora bien, explicar exhaustivamente que significa "conocimiento" no parece posible en el estrecho margen de esta investigación. No obstante, si parece posible desarrollar una comprensión básica de aquello que significa que X "conoce". En lo que sigue, se introducirá sintéticamente ciertos aspectos de la epistemología analítica con el objetivo de reformular el significado del término "conocimiento".

\footnotetext{
${ }^{6}$ ETCHEBERRY (1997), p. 278; POLITOFF (1997), p. 526; GARRIDO (2003), p. 205; COUSO (2006), p. 153; POLITOFF et al. (2003), p. 294; KÜNSEMÜLLER (2001), p. 180.

7 Véase el clásico ensayo de DAVIDSON (1963), pp. 685-700 y más en general WILAND (2010), pp. 1-13.

${ }^{8}$ Se entiende aquí el término "representacional" en un sentido deflacionista, es decir, de una manera suficientemente amplia como para considerar representacional algunos aspectos de aquellos considerados no-representacionales en la moderna ciencia cognitiva, siguiéndose aquí a HUTTO (2015), pp. 63-83.

${ }^{9}$ Ciertamente hay muchas controversias aquí, por ejemplo, entre interpretaciones cognitivistas de los estados de "deseo" como los desarrolla QUINN (1993), pp. 3-18, versus interpretaciones no-cognitivistas como los desarrolla VELLEMAN (1992), pp. 10-13. Para una visión panorámica véase SCHROEDER (2015).
} 
Es una posición estándar en la epistemología analítica contemporánea entender el significado de "conocer" en términos de afirmar que $X$ conoce cuando $X$ tiene una creencia verdadera justificada ${ }^{10}$. Consideremos la afirmación " $X$ sabe que $P$ " ( $X$ sabe que $P$ es el caso 0 que $P$ es verdadero) en términos de una creencia verdadera justificada. Esta afirmación debería satisfacer tres condiciones:

(1) $P$ es verdadero

(2) $X$ cree que $P$ es verdadero ( $X$ cree que (1) es verdadero)

(3) La creencia de $X$ está justificada (la creencia (2) debe estar basada en razones)

La primera condición tiene por objetivo rechazar la idea que $X$ puede "conocer" $P$ cuando $P$ no es verdadero. Digamos que $P=$ la heladería está en la esquina. Para poder afirmar que $X$ "sabe" o tiene "conocimiento" que $P$, es decir, que la "heladería está en la esquina", tiene que ser verdadero que la heladería está en la esquina. Si la heladería no está en la esquina, no puede ser correcto que $X$ sabe que $P$. La segunda condición tiene por objetivo asegurar que el sujeto en realidad tiene la creencia que $P$, es decir, cree que "la heladería está en la esquina", porque no parece tener mucho sentido adscribir a $X$ " $X$ sabe que $P$ " si $X$ no cree que $P$ cuando, por ejemplo, $X$ nunca ha estado en el centro de la ciudad que ahora visita. En efecto, en este último caso si $X$ no sabe que la heladería está en la esquina, entonces no puede tener dicha creencia. La tercera condición tiene por objetivo asegurar que $X$ tiene algún tipo de razón para creer que $P$ es verdadero, porque para poder afirmar que $X$ sabe que $P$ debe estar justificado en su creencia que "la heladería está en la esquina". De este modo, no tendría mucho sentido adscribir a X "X sabe que P" si la justificación de la creencia de X es que "vio una estrella fugaz" en el cielo y, entonces, "supo" que la heladería estaba en la esquina. Nótese que esta conclusión es correcta (que X no "conoce") incluso si $\mathrm{P}$ es verdadero (se satisface 1 , porque la heladería está en la esquina) y $X$ cree que $P$ es verdadero (se satisface 2 , porque $X$ cree que la estrella fugaz le "dijo" que la heladería estaba en la esquina). Ahora bien, luego de este excesivamente breve sumario epistemológico ${ }^{11}$ veamos si se puede perfilar más precisamente aquello que debería entenderse por conocimiento en el derecho penal o si, por el contrario, la introducción de explicaciones epistemológicas parece problemática.

En una primera aproximación esta noción de conocimiento efectivamente parece problemática. En efecto, si se entendiera el conocimiento en el derecho penal como creencia verdadera justificada parecería muy difícil establecer que $\mathrm{X}$ obra con dolo y sentaría las bases para excluir del castigo penal, al menos, los casos de dolo eventual. Veamos el problema más de cerca. Digamos que $X$ despliega un curso de conducta y acepta que la consecuencia de su comportamiento puede involucrar la muerte de $\mathrm{Y}$. $\mathrm{X}$, aceptando la posible consecuencia de su conducta la continúa realizando de modo que ella concluye con la muerte de $Y$. El problema es que a pesar que $X$ haya aceptado como posible un resultado, ello no significa que $X$ cree que de su conducta se puede seguir como resultado la muerte de $Y$ porque " $X$ acepta que $P$ " no es equivalente a "X cree que $P$ ". En efecto, puede haber aceptación sin que exista creencia ${ }^{12} \mathrm{y}$, si puede haber aceptación sin creencia, entonces no se podría satisfacer la condición (2). Es decir, si se sigue esta línea argumentativa ello debería conducir a la conclusión que en los casos de aceptación sin creencia $X$ actúa sin dolo, porque $X$ no satisface las condiciones mínimas para establecer que hay conocimiento.

El conocimiento, además, requiere de la posesión del sujeto de una creencia específica actual ${ }^{13}$ que en este caso $X$ no necesariamente tiene $y$, si no la tiene como en algunos casos de dolo eventual, entonces tampoco habría conocimiento. No obstante, estos dos problemas se pueden corregir si se flexibiliza la noción de "creencia" de modo que abarque la aceptación y no exigiéndose que esta sea actual. De este modo, a pesar que no ocurra una creencia actual en el

\footnotetext{
${ }^{10}$ DANCY (1985) p. 3; STEUP (1996), pp. 1-2; AUDI (1998), p. 2; WILLIAMS (2001), pp. 16-17; PRITCHARD (2016) pp. 3-4.

${ }^{11}$ En el cual ni si quiera se ha considerado los casos de Gettier. Para una explicación detallada de estos casos véase HETHERINGTON (2016), capítulo 1.

${ }^{12}$ COHEN (1992), capítulo 1, pp. 1-27.

${ }^{13} \mathrm{Al}$ menos para la literatura que entiende el conocimiento como un estado mental, véase NAGEL (2013), pp. 273-308.
} 
sujeto $X$, sería posible adscribírsela si hay evidencia para considerar que (en base a lo que hizo $X$ ) esta podría encontrarse justificada (3). Esta flexibilización de la noción de creencia no parece proceder respecto del término conocimiento y por ende aparece lo apropiado del término "creencia"14. Esto sugiere que es plausible entender que algo menos exigente de satisfacer que el "conocimiento" es suficiente para la responsabilidad penal, concediendo al término "creencia" un valor central ${ }^{15}$. En suma, la literatura epistemológica analítica permite articular de una forma más rigurosa las exigencias que satisfacen la culpabilidad como "conocimiento de la antijuridicidad de la conducta": esta oración hace referencia a ciertas formas de creencia que, bajo ciertas condiciones evidenciales, pueden adscribírsele a una persona y cuyo objeto es una determinada forma de comportamiento que tiene la característica de ser antijurídica.

\section{Conocimiento de la antijuridicidad como creencia razonable}

Antes de examinar críticamente la doctrina chilena es necesario reformular que es una conducta dolosa y que es una conducta culpable de acuerdo a la terminología introducida en la sección precedente. Esto puede hacerse incluyendo dos sub-condiciones a (2):

(2.1) $X$ cree que realiza una conducta determinada

(2.2) $X$ cree que la conducta que realiza es antijurídica

La afirmación (2.1) es una forma alternativa (pero equivalente) de entender una conducta dolosa y que se apega a la sustitución del término "conocer" por el término "creer" propuesto previamente. Cuando $X$ satisface la condición (2.1) esto significa que $X$ cree (sabe) que está realizando una determinada acción $A$, es decir, que $X$ despliega una conducta dolosa. Por ejemplo, si $X$ "cree" que realiza la acción $A$ cuando $A$ consiste en la acción de disparar a $Y$, entonces esto es lo mismo que afirmar que $X$ tiene dolo cuando hace $A$, es decir, que $X$ actúa dolosamente cuando dispara a Y. En contraste, la capacidad cognitiva relevante para la culpabilidad es la condición (2.2) y cuyo objeto no es solamente una "conducta" sino una "conducta antijurídica" ${ }^{16}$. Estas proposiciones y sus implicancias para el error de tipo del inimputable se exploran a continuación.

Teniendo presente la distinción entre (2.1) y (2.2) examinemos como la doctrina chilena persigue caracterizar el tipo de creencia que tiene que estar presente para afirmar que (2.2) es correcta. Para satisfacer el juicio de culpabilidad la creencia de $\mathrm{X}$ debe tener por objeto una conducta caracterizada como antijurídica, pero la doctrina caracteriza el contenido de esta creencia de manera diferente. Mientras que un sector que se asociará con Cury entiende que la conducta culpable (2.2) debe haber sido previamente establecida como dolosa ${ }^{17}(2.1)$, otro sector que se asociará con Etcheberry entiende que la conducta culpable (2.2) es posteriormente caracterizada como dolosa (2.1). Es decir, este último sector de la doctrina chilena $^{18}$ considera que establecer que una conducta es dolosa supone haber establecido previamente que se trata de una conducta culpable. Esto tiene importantes consecuencias para

\footnotetext{
${ }^{14}$ Esto en ningún caso implica que en muchas ocasiones el sujeto activo del delito ciertamente "conoce" en sentido epistemológico. Se prefiere el término "creencia" porque es menos estricto para establecer las condiciones que son suficientes para dar por establecido el dolo.

${ }^{15}$ Esta interpretación se alinea con las teorías cognitivas del dolo, pero sugiere que estas pueden refinarse sustituyendo la exigencia de "conocimiento" por la exigencia de posesión de "creencias" por parte de del sujeto. Sobre estas teorías véase RAGUÉS (2004), pp. 15-16 y SOTOMAYOR (2016), pp. 681-683.

${ }_{16}$ Mañalich ha argumentado que el conocimiento de la antijuridicidad de la conducta se encuentra en una relación de superveniencia con el dolo, lo que significa que (2.2) depende del establecimiento de (2.1). En otras palabras, afirmar que $X$ satisface (2.2) supone que X sabe que conducta está realizando, véase MAÑALICH (2011), pp. 99-102. Si bien Mañalich está en lo correcto, no parece que la mejor forma de caracterizar la relación ente (2.1) y (2.2) sea en términos de relaciones de superveniencia. La razón es que la relación de superveniencia es una relación fundamentalmente reductiva. Caracterizar la relación entre (2.1) y (2.2) en términ os de superveniencia tendría la implicancia de afirmar que el conocimiento de la antijuridicidad de la conducta podría reducirse al conocimiento de la conducta. Después de todo, entender esta relación en términos de superveniencia, es precisamente permitir la explicación de lo que superviene en términos de la base subveniente.

17 Aquí podemos encontrar a los siguientes autores: CURY (2005), p. 385; COUSIÑO (1975), p. 64; BUSTOS (1994) pp. 505-513; NÁQUIRA (1998), pp. 123 y 322; GARRIDO (2003), pp. 95 y 253; VARGAS (2010), pp. 69 y 127; MAÑALICH (2011), p. 103.

${ }^{18}$ Aquí podemos encontrar a los siguientes autores: ETCHEBERRY (1997), p. 275; LABATUT (1989), pp. 87-90; POLITOFF et al. (2003), p. 245; NOVOA (2005), p. 402.
} 
la procedencia de las medidas de seguridad en el caso del error de tipo del inimputable. En particular, como se verá, la respuesta que esta posición elabora adopta una comprensión muy poco plausible de la exigencia de "hecho típico" prevista en el art. 455 del CPP.

En el sistema que se ha asociado a Etcheberry, negar que una determinada conducta es dolosa no significa negar necesariamente que dicha conducta es susceptible de ser calificada como peligrosa (en el sentido de poder hacerse acreedora de una medida de seguridad), porque la descripción de una conducta dolosa solo aparece analíticamente con posterioridad a su descripción como conducta culpable ${ }^{19}$. Si la prioridad analítica la tiene la descripción de una conducta culpable, entonces el parámetro para evaluar si una conducta puede ser objeto de una medida de seguridad es todavía viable. Entonces, el sistema de Etcheberry permite que previo a que la conducta sea descrita como dolosa proceda la determinación de si es o no peligrosa, lo que corresponde evaluarse naturalmente en la categoría de la culpabilidad. Esto, sin embargo, crea un serio problema para esta posición y pone serias dudas sobre su plausibilidad. Veamos esto más de cerca considerando el caso de $X 1$ quien mata a $Y$ con un cuchillo porque cree que está faenando una vaca, es decir, X1 motivado por un desorden mental actúa con error de tipo. El art. 455 del CPP establece que se puede aplicar una medida de seguridad solo si X1, además de ser considerado peligroso, ha realizado "un hecho típico y antijurídico". ¿Qué cabe entender aquí como un hecho típico? Hemos adelantado que si se entiende por "típica" una conducta que debe satisfacer exigencias objetivas (imputación objetiva) como subjetivas (dolo o culpa), ello significa que Etcheberry debe aceptar que de alguna manera se satisfacen estas últimas aún cuando en este caso X1 actúa sin dolo. Es decir, si la tesis de Etcheberry admite la aplicabilidad de las medidas de seguridad para X1, entonces se ve forzada a adoptar una interpretación del art. 499 del CPP como un requerimiento exclusivamente objetivo.

Si la exigencia que una conducta sea típica se interpreta exclusivamente de forma objetiva si podría satisfacerse el requerimiento del art. 455 del CPP. Es decir, si se entiende que no es necesario que el inimputable actúe dolosamente, entonces cuando actúa con error de tipo si sería posible aplicar una medida de seguridad. Sin embargo, esta argumentación cuyo resultado pudiera parecer aceptable es criticable por tres razones. Primero, porque hay muchas disposiciones cuyos hechos operativos no pueden ser subsumibles en el tipo sin recurrir a criterios subjetivos (como los delitos contra la propiedad). Segundo, porque no hay ningún argumento que explique o justificación que permita suprimir estas exigencias subjetivas cuando se trata de una persona inimputable. La exigencia de dolo o imprudencia son requerimientos del principio de culpabilidad que prohíbe la responsabilidad penal objetiva, por ende, para ser plausible, debería explicitarse las razones por las que supuestamente el ordenamiento penal puede permitirse no cumplir estas exigencias cuando se trata de una persona inimputable. Tercero, incluso si se suministran dichas razones, habría que explicitar el porqué debería considerarse esa interpretación discriminatoria como una interpretación admisible del principio de culpabilidad. En conclusión, estos tres problemas conducen a estimar inadecuada la postura asociada a Etcheberry y a considerar, por ende, la doctrina asociada a Cury.

Ahora bien, esta última doctrina si bien parece ser más plausible, porque considera que la afirmación de (2.1) precede a la afirmación de (2.2), no está exenta de problemas cuando se tiene presente el caso de X1. Si se considera que la actuación dolosa está referida a la conducta que X1 está realizando, ¿̇sería aceptable concluir que actúa con error de tipo aquel que no cree que conducta realiza porque tiene un desorden mental? Si X1 actúa sin dolo porque tiene un desorden mental eso significa que la conducta que X1 realiza es atípica y, entonces, la conclusión debería ser que en estos casos no es aplicable una medida de seguridad porque el hecho no es "típico". Como se ha visto, en el art. 455 del CPP el término "típico" exige la concurrencia de estos elementos subjetivos. En consecuencia, si una persona con un desorden mental actúa sin satisfacer dichas condiciones, como X1, entonces no puede aplicársele una medida de seguridad. Esta conclusión ha sido sugerida por los profesores Artaza y Carnevali

${ }^{19}$ Véase VARGAS (2016), pp. 323-351. 
[...]se debe concluir que resulta razonable sostener que, en determinadas situaciones en que un sujeto presenta ciertos trastornos mentales que no le permitan alcanzar el conocimiento requerido por el tipo penal, no podría atribuirse un comportamiento doloso al mismo... Como se pudo apreciar, parte de la práctica judicial reconoce que el juicio de adscripción del dolo, como atribución de conocimiento, se hace tomando en cuenta las circunstancias personales del agente, incluyendo en estas la individualidad del sujeto. Así las cosas, pareciera que en el proceso conocido como de "imputación subjetiva" no bastaría el ponerse en lugar del autor sin considerar su capacidad para conocer el significado de su comportamiento, y que este realiza los elementos de un tipo penal en particular ${ }^{20}$.

El problema de la tesis asociada a Cury es que no parece apropiado sostener que en los casos de error de tipo, cuando este es generado por un desorden mental, no pueda aplicarse nunca una medida de seguridad ${ }^{21}$. En efecto, parece que en estos casos al menos debería poder evaluarse la procedencia de una medida de seguridad, no solo porque el sujeto puede ser peligroso, sino porque para nuestras prácticas de responsabilidad una persona que comete un delito causado por un desorden mental es posiblemente el tipo de persona apta para ser destinataria de este tipo de medidas ${ }^{22}$. La posición asociada a Cury, no obstante, no permite esta evaluación porque la decisión afirmativa sobre el error de tipo la precluye. Si bien parece correcto que al menos pueda evaluarse racionalmente la procedencia de una medida de seguridad para el caso de $\mathrm{X} 1 \mathrm{y}$, por ende, discutir en un proceso penal su posible procedencia, no es claro cual podría ser la razón que lo justifique. Parece necesario indagar más de cerca en que es la inimputabilidad, porque posiblemente la respuesta se encuentre en el tipo de incompetencia que un inimputable presenta y como esta se relaciona con las exigencias de conocimiento que forman parte de las condiciones de la aplicación justificada de la medida de seguridad. Para ello examinemos la siguiente proposición (2.2.1):

(2.2.1) $\mathrm{X}$ no cree que la conducta que está realizando es antijurídica por causa de un desorden mental

(2.2.1) debe ser comprendida como una proposición que expresa la incapacidad cognitiva clave para comprender porque una persona puede ser considerada inimputable. Más específicamente, es una incapacidad de razonar que involucra la afectación del proceso de formación de creencias del agente para establecer las diferentes alternativas de satisfacción de sus deseos en los planes que formula sobre su futuro. He sostenido en un trabajo previo ${ }^{23}$ que esta incapacidad cognitiva implica que la persona normalmente no puede desempeñarse en el mundo social de forma más o menos racional y, por lo tanto, impide afirmar que el sujeto es capaz de guiarse por las razones del derecho penal. La explicación de esta insuficiente competencia en las interacciones sociales residiría en la existencia de un desorden mental. Es natural entender que las creencias, como se ha visto, son necesarias para establecer planes para el futuro porque nos permiten coordinarnos con nuestro medio-ambiente físico y social. Por lo tanto, si esta capacidad se encuentra afectada por un desorden mental que, por ejemplo, hace que $\mathrm{X} 1$ crea que cuando mata a Y está faenando una vaca, aparece que dicha afectación en el proceso normal de formación de creencias le impide al sujeto manejarse de manera competente, porque de no haber tenido dicha incapacidad habría comprendido que $\mathrm{Y}$ era una

\footnotetext{
${ }^{20}$ ARTAZA y CARNEVALI (2018), p. 33.

${ }^{21}$ Ciertamente debe una buscarse una respuesta en este sentido pero que desde luego no tenga las implicancias de las tesis defendidas por Fernando Molina y Silva Sánchez. Molina al sostener que es poco plausible comprender que un hecho que es realmente lesivo pueda dejar de ser antijurídico por faltar un elemento subjetivo, considera que el caso del inimputable es equivalente al del imputable que actúa con error de tipo y, en consecuencia, considera que a ambos podría aplicarse una medida de seguridad, MOLINA (2008) p. 144. Silva Sánchez, por otro lado, considera que la antijuridicidad de las medidas de seguridad es diferente al de la pena y que se satisface con "la lesión objetivamente típica y no justificada de un bien jurídicopenal" y, con ello, se compromete con los mismos problemas que aquejan a la tesis de Etcheberry, SILVA (2003), p. 127.

${ }^{22}$ Ver FERNÁNDEZ (2021a), pp. 13-16.

${ }^{23}$ FERNÁNDEZ (2021a), pp. 3-6.
} 
persona y por lo tanto, de haber podido guiarse por las razones del derecho penal, no se encontraría en una situación susceptible de ser objeto de una medida de seguridad ${ }^{24}$.

Desde este punto de vista aparece que en tanto competencia "social" el criterio que determina la competencia es en gran medida un estándar objetivo. Es decir, en la determinación de quien no tiene estas capacidades de formar creencias competentes, opera un estándar de objetividad que evalúa las capacidades que consideramos necesarias para desempeñarnos en nuestro mundo social. Esto tiene implicancias importantes para como debemos interpretar (2.2), es decir, el tipo de creencia que es apropiada para afirmar que $X$ tiene conocimiento de la antijuridicidad de su conducta. En efecto, si es correcto que hay un estándar objetivo que marca las interpretaciones correctas de aquellas interacciones sociales incompetentes, entonces (2.2) no puede significar atribuir un significado que se determina exclusivamente de acuerdo a lo que cada persona cree, piense u opine. De esto se sigue una consecuencia importante: quien tiene un desorden mental no puede aducir como razón para comprender la antijuridicidad de su conducta de manera diferente que sus creencias son diferentes ${ }^{25}$. En otras palabras, no puede introducir su propio punto de vista como parte de la justificación de su creencia. Esta exigencia se denominará "razonabilidad" de la creencia.

En el esquema de condiciones propuesto la exigencia de razonabilidad es inherente a la satisfacción de la condición (3), porque esta exige recurrir a criterios públicos donde $X$ al ejercitar sus competencias sociales demuestra que los satisface o $\mathrm{no}^{26}$ y que en tanto exigencia contenida en (3), como veremos, se aplica a las condiciones (2.1) y (2.2). Algo semejante se plantea por la doctrina chilena y comparada sobre el tipo de creencia que sería exigible esperar de una persona sobre su comportamiento. En efecto, la noción de "valoración paralela en la esfera de lo profano" persigue de alguna manera objetivizar la comprensión del sujeto, de modo de seleccionar el tipo de creencias adecuadas para considerar que se satisfacen las condiciones de responsabilidad penal. La noción de "conocimiento del profano" objetiviza la exigencia de creencia recurriendo a como un no-experto podría tener dicha creencia, pero aquí aparecen ciertas diferencias entre esta y la noción de razonabilidad. Primero, la "valoración paralela" parece apelar a una comprensión estadísticamente relevante de una determinada conducta que sería la conducta del "profano", pero en la razonabilidad no se apela a una conducta que es estadísticamente relevante, sino a la aplicación correcta de un concepto y que manifiesta la adquisición de una competencia social ${ }^{27}$.

\footnotetext{
${ }^{24}$ Este juicio contrafáctico es útil, porque al ayudar a identificar el caso del sujeto que de encontrarse en una situación en que el proceso de formación de creencias es normal y que no habría ejecutado dicha acción, permite aislar el desorden mental como aquella causa que explica en definitiva el hecho. Ahora bien, de ser el caso que de todas formas se habría formado el deseo de matar al vecino y seleccionado los medios apropiados logrando conseguir su propósito, este resultado si podría ser considerado como un resultado "suyo" y como una expresión de agencia, lo que es precisamente lo que falta cuando aquel se explica por la existencia de un desorden mental.

${ }^{25}$ Algo diferente sostiene BUSTOS (1987), p. 299, en el sentido que afirma que, si hay una gran variedad de racionalidades, incluyendo la de quien padece un desorden mental, entonces esta debe ser respetada por razones morales fundadas en el subjetivismo ético. Fernández ha sostenido recientemente que no pueden considerarse en este tipo de casos a los miembros de pueblos indígenas FERNÁNDEZ (2021b), pp. 10-14.

${ }^{26}$ Visto así, por evidencia no se entiende aquí solamente la estimulación de receptores sensitivos localizados en nuestros cuerpos, como QUINE (1968), p. 75, porque no solo debemos considerar como evidencia aquello que "puede hacer una diferencia en lo que es justificado creer" como afirma KELLY (2014), es decir, aquellos objetos o proposiciones que justifican nuestras creencias, sino que "evidencia" debe ser entendida aquí en sentido lo suficientemente amplio como para abarcar el proceso por el cual arribamos a dicha evidencia.

${ }^{27}$ Alex van Weezel ha utilizado una noción de razonabilidad similar, sin embargo, la siguiente propuesta es diferente. Para van Weezel $X$ es competente cuando $X$ puede ser considerado culpable, pero la noción de competencia social de la propuesta apela a las capacidades prácticas y lingüísticas que posibilitan que una persona adopte la posición de participante en sus prácticas sociales. Desde esta perspectiva, $X$ puede ser competente porque es socialmente competente, mientras que van Weezel parece considerar que la competencia social es independiente de la competencia que determina que una persona sea culpable para el sistema de justicia penal. Más allá de esto, es difícil precisar que entiende el autor cuando utiliza el término "razonable". Van Weezel parece afirmar que cuando X actúa de manera "razonable" esto equivale a afirmar que X no es competente y, por ende, no es culpable de un determinado delito por no haber, por ejemplo, conocido que lo que hacía era antijurídico. Sin embargo, en su interpretación también introduce elementos volitivos (VAN WEEZEL (2008), p. 41), lo que dificulta comprender su uso del término "razonabilidad". Hay un último aspecto de la propuesta del autor que la diferencia de la propuesta que se desarrolla en este trabajo. Van Weezel considera que el objeto de la creencia es irrelevante para establecer la razonabilidad, pero para la presente propuesta dicha
} 
Segundo, y esto es lo crucial, la objetivización desarrollada por la doctrina se plantea exclusivamente como una exigencia dentro de la condición $(2.1)^{28}$. Es decir, en la valoración paralela la creencia no va referida a lo que hace reprochable la conducta. En contraste, la objetivización de la exigencia de razonabilidad no está referida exclusivamente a (2.1), sino a las condiciones que debe reunir tal conducta para ser "comprendida" como competente por el resto de los participantes en la respectiva práctica social ${ }^{29}$. Esta no es una diferencia sutil. La razonabilidad se refiere a la comprensión de un determinado hecho social y ello solo puede determinarse "internamente" por la misma colectividad. La objetivización que se está considerando persigue hacer explícito que en la caracterización de " $X$ cree que la conducta que realiza es antijurídica" hay involucrado un punto de vista social que se considera autorizado no solo para establecer dicha caracterización como razonable, sino además como condición para establecer la competencia social de un sujeto y como requerimiento para la responsabilidad penal. Esto no es sino apelar al hecho que no solo hay un estado que aplica una pena, sino una comunidad política para la cual definir una conducta como reprochable y frente a la cual la pena o medida de seguridad es una respuesta apropiada, es algo susceptible de legitimación política. Ahora podemos formular esta exigencia de razonabilidad en el esquema que se ha propuesto adicionando una sub-condición a (3):

(3.2) la creencia (2.2) es una creencia razonable

\section{La creencia razonable, el error de tipo del inimputable y el error de prohibicion del imputable}

En esta sección final se examinará con mayor profundidad la condición (3.2) en el esquema analítico de esta investigación. Una "creencia razonable" 30 que satisface (3.2) es una creencia que satisface (1)-(3.1), lo que se puede precisar más detalladamente dándole contenido penal a P1:

P1 = hay una descripción de una conducta prohibida que es caracterizada por el sistema jurídico como penalmente prohibida y la conducta de $X$ es subsumible en el supuesto de hecho de la disposición penal que la prohíbe

De esta manera cuando afirmamos que P1 "es verdadero" afirmamos que la conducta que $X$ realiza es constitutiva de delito, por ejemplo, digamos que $X$ dispara a $Y$ cometiendo un delito de homicidio. Con respecto a la terminología epistemológica sugerida, si afirmamos respecto de un sujeto $X$ que este satisface (3.2), porque tiene una creencia razonable sobre la antijuridicidad de su conducta, entonces también afirmamos que P1 es verdadero, es decir, que se satisface (1) porque $X$ ha cometido un delito de homicidio. Adicionalmente, también satisface (2), es decir, $X$ cree que $P 1$ es verdadero. $X$ también satisface (3), es decir, $X$ tiene al momento de realizar su conducta una creencia justificada. En otras palabras, $X$ tiene algún tipo de evidencia que haga plausible suponer que otros/as en su situación hubieran creído que P1 era verdadero. Naturalmente, si $X$ cree que tiene una pistola cargada en la mano no habría problema alguno, pero incluso si no tiene esa creencia "actual", en ciertos casos, nada impediría inferir que " $X$ cree que si dispara puede matar a $Y^{\prime \prime}$ a partir de lo que $X$ hizo. Como se ha visto, no parece ser

referencia es importante porque considerar los diferentes objetos de las creencias es lo que permite distinguir entre las condiciones (2.1) y (2.2).

28 POLITOFF et al. (2003), p. 294; CURY (2005), p. 308; ROXIN (1997), p. 460; KINDHAUSER (1999), p. 155 considera que esto sería relevante solo para la culpabilidad.

${ }^{29}$ Sobre la noción de "participante" véase STRAWSON (2008), p. 10.

${ }^{30}$ Cabe notar que la propuesta no entiende la "creencia razonable" como aquella "creencia que es razonable tener" porque esta última enfatiza el tipo de creencia que un sujeto debería tener, por ejemplo, para poder actuar moralmente. Tampoco identifica razonabilidad con racionalidad, véase KALLESTRUP (2020), p. 9. Ahora bien, podría pensarse que lo que se requiere es un tipo de creencia que responda a lo que el sujeto efectivamente cree, pero no es esta la interpretación que se propone. "Creencia razonable" se refiere no a lo que el sujeto efectivamente cree, sino a las creencias que son socialmente correctas de tener. La propuesta interpreta el término "creencia razonable" objetivamente. Para una comprensión opuesta y estrictamente subjetivista de creencia razonable véase SMITH (2011), pp. 7-9. 
necesario que la evidencia sea una que solo si toda persona hubiera tomado como premisa hubiera inferido que P1 era verdadero. Para que a X pueda adscribírsele justificadamente la creencia (2), es decir que satisfaga (3), no se requiere un tipo de evidencia que solo Dios podría tener, sino el tipo de evidencia que es exigible a personas que toman parte en nuestras interacciones sociales como seres que tienen información imperfecta sobre el mundo. Ello significa que si $X$ afirma (luego de haber disparado y matado a $Y$ ) "yo creí que la pistola era de plástico", pero de la evidencia disponible y probada en juicio se concluye que la pistola era de metal, pesada y aparentaba para una persona común y corriente una pistola real, entonces a pesar que sea efectivo que $X$ creyó que la pistola era de plástico dicha creencia no constituirá obstáculo para establecer, al menos, dolo eventual y por ende debería tenerse por satisfecha, adicionalmente, la condición (2.1).

Dejemos de lado por el momento las exigencias relativas al dolo, es decir si se cumple o no (2.1) y consideremos el siguiente caso: $\mathrm{X} 2$ mata a $\mathrm{Y}$ porque (causado por un desorden mental) ha escuchado palabras de "dios" quien le ha ordenado que mate a $Y$ y considérese la condición (2.2). En este caso $X 2$ tiene un desorden mental que causa una creencia que lo inhabilita a cumplir la condición (2.2) y parece entonces que X2 no podría satisfacer la condición (3.2). En efecto, cuando $X 2$ tiene un desorden mental que lo priva de las capacidades cognitivas necesarias para la culpabilidad, debe concluirse que $X 2$ no tiene la capacidad para creer que su conducta es antijurídica (3.2). En efecto, X2 no tiene esta capacidad porque tiene creencias irrazonables. La caracterización de la creencia como irrazonable es fundamental aquí, porque podría ocurrir el siguiente caso: $X 3$ cree que $Y$ es un terrorista y le disparó (y mató) antes que hiciera explotar un edificio. Si dicha creencia es considerada razonable (porque X3 vio que $Y$ dejó en el suelo una mochila sospechosa y le pareció escuchar que $Y$ hablaba con $Z$ para detonar el edificio), entonces ello significa que X3 si bien satisface (2.1) no satisface (2.2), porque como luego se demuestra en el juicio $Y$ no era un terrorista y lo que $Y$ habló con $Z$ decía relación con limpiar el edificio. No obstante, no parece plausible concluir lo mismo si X2 mató a Y porque escuchó a dios que le decía "mata a Y" y ello fue causado por un desorden mental. De hecho, es indicativo de la diferencia la posible respuesta del derecho penal: X3 podría ser exculpado (si el error se estima invencible) mientras que $\mathrm{X} 2$ podría ser candidato a una medida de seguridad.

Trasladémonos ahora a otro caso de un imputable que actúa con error de prohibición para identificar ciertas semejanzas con el caso del inimputable, porque este es el paso previo para comprender como opera la exigencia de razonabilidad a nivel de dolo. El caso ha sido propuesto por Juan Pablo Mañalich

[...]si un sujeto con motivaciones racistas dispara un arma de fuego, letalmente, sobre una persona perteneciente a un determinado grupo étnico, en la creencia de que no se trataría de "otro", en el sentido de la tipificación del homicidio, entonces para determinar si el hecho le es imputable -en el nivel del injusto (subjetivo)- a título de dolo hay que preguntarse si, bajo la suposición de que el autor hubiera conocido perfectamente el sentido y alcance de la prohibición del homicidio -que es precisamente lo que en el caso no se da-, su representación de las circunstancias fácticas objetivamente dadas le habría posibilitado formarse la intención de evitar el disparo, en pos del seguimiento de la prohibición. Y la respuesta, entonces, es ciertamente afirmativa. La creencia errónea del autor constituye un error conceptual (o de sentido) -en el cual se expresa, por lo demás, un muy serio error moral de su parte-, que sólo podría llegar a tener relevancia para la imputación en la medida en que efectivamente implicase una falta de conocimiento de la antijuridicidad del hecho, es decir, a modo de un error de prohibición ${ }^{31}$.

De acuerdo a Mañalich si un sujeto Z1 con motivaciones racistas considera que mata a una persona inferior, entonces $\mathrm{Z} 1$ actúa con dolo pero podría apreciarse un error de prohibición en ciertos casos. Digamos que Z1 cree que Z2 es una persona para efectos del delito de homicidio, pero que $\mathrm{Z} 1$ cree puede matar a $\mathrm{Z} 2$ porque $\mathrm{Z} 2$ es una persona inferior que debe tratar

${ }^{31}$ MAÑALICH (2011), p. 105. 
con excesivo respecto a Z1. En el caso concreto Z2 no trata con este excesivo respeto a Z1 y como consecuencia Z1 mata a Z2. ¿Porqué podría pensarse que Z1 actúa con error de prohibición? Podría haber un error en la medida que se considere que Z1 cree erróneamente que un determinado predicado, a saber, "ser persona con derecho a la vida" no se aplica a un objeto, en este caso a $Z^{32}$. Adicionalmente digamos que $Z 1$ vivió y creció en una familia Nazi donde le enseñaron que aquellos como $Z 2$ son personas inferiores que no tienen derecho a la vida como las personas como Z1. Bajo este nuevo contexto, si Z1 cree honestamente que Z2 es una persona inferior porque así le enseñaron y así aprendió, entonces si bien Z1 actúa con dolo de matar, en el entendido que mata de acuerdo a las disposiciones sobre homicidio que requieren que el sujeto crea que a quien priva de vida es una persona, Z1 podría, no obstante, actuar con un error de prohibición. Pero, ¿̇sería correcto entender que actúa Z1 actúa con error de prohibición? Mañalich podría responder ${ }^{33}$ que si Z1 hubiera conocido correctamente el sentido del término "persona con derecho a la vida" se podría haber formado un motivo de evitar matar a Z2. En efecto, si Z1 hubiera entendido que Z2 era una persona con derecho a la vida, que al matar a Z2 mataba a una persona con tal derecho y que por ende podría ser sancionado por las disposiciones del homicidio, entonces Z1 podría haberse formado una intención (de segundo orden) de evitar matar a Z2.

Ahora bien, la (posible) afirmación de Mañalich todavía no responde a una pregunta fundamental: ¿'es correcto asumir que Z1 estaba en un "error"? En efecto, el nazi honesto Z1 podría perfectamente afirmar de acuerdo a su ideología que los sujetos Z2 son personas inferiores y que, por lo tanto, al estar honestamente convencido que $Z 2$ es una persona inferior, no habría podido formarse el motivo de evitar matar a Z2 cuando Z2 no lo trató con el respeto que Z1 creía merecer ${ }^{34}$. En este caso Z1 no se habría formado una intención de evitar y quizás de manera invencible dado su contexto de crianza y aprendizaje. Entonces aparece el problema crucial, ¿ basta que Z1 no haya podido evitar formarse la intención de matar a Z2 para afirmar que Z1 no cree que su conducta es antijurídica? Si se trata de una cuestión meramente fáctica, la respuesta debería ser positiva y podría apreciarse la concurrencia de un error de prohibición. Esta conclusión, no obstante, parece incorrecta, pero para explicar esta incorrección debemos acudir a otra exigencia que Z1 debe cumplir, a saber, aquello que requiere (3.2): incluso si Z1 no pudo formarse la intención de evitar matar a Z2 si su creencia no es razonable, entonces no derrota la presunción de conocimiento de la antijuridicidad de su comportamiento ${ }^{35}$. Es decir, Z1 no actúa con error de prohibición.

La exigencia de razonabilidad, como puede verse, no apela exclusivamente a las condiciones de verdad de una determinada sentencia o a las propiedades que efectivamente se predican de un objeto. Esto significa que no es suficiente para la comprensión correcta del término "persona" conocer cuales son sus propiedades y cuales son sus condiciones de verdad porque, en rigor, no se trata solamente de atribuir a una persona un estado intencional, sino de establecer si dicho estado intencional es razonable o no y para establecer esto se requiere precisar si de acuerdo a las prácticas sociales en que el proceso penal tiene lugar, Chile, tal creencia es razonable ${ }^{36}$. Como la creencia de Z1 no es razonable (en Chile), Z1 si puede ser condenado por homicidio negando la posibilidad de un error de prohibición. Enfatizar

\footnotetext{
32 Esta es la respuesta de Kindhaüser y que denomina como "error de sentido", véase KINDHAÜSER (1999), p. 145 y KINDHAUSER (2019), p. 135.

${ }^{33}$ Sigue aquí a KINDHAÜSER (1999), pp. 155-156.

${ }^{34}$ Van Weezel desarrolla un concepto de error ciertamente diferente del de Mañalich, pero ambos recurren a la idea de la persona "fiel al derecho" (2008, p. 39), es decir, aquel que podría haberse motivado y habría podido, entonces, modificar su intención y abstenerse de actuar típicamente. En la medida que parta de esta premisa compartida, van Weezel debería llegar a la misma conclusión que Mañalich: la conducta del nazi honesto podría eventualmente excusarse en la medida que revele "un déficit que de alguna forma no es imputable al autor, sino a la sociedad misma (deficiente socialización del sujeto o de la norma en cuestión)." VAN WEEZEL (2008), p. 41. En efecto, si Z1 tiene las creencias que tiene por "deficiente socialización", entonces no es respo nsable de las creencias que lo llevan a actuar y a privar a Z2 de su vida. Ciertamente, parece posible refutar esta conclusión adhiriendo a otro tipo de premisa, pero lo que aquí interesa subrayar es lo problemático de la premisa misma.

35 Podría afirmarse que la creencia irrazonable no "derrota" la presunción simplemente legal de culpabilidad.

${ }^{36}$ Para una crítica general al concepto de "condiciones de verdad" para una teoría del significado, véase LYCAN (2019), pp. 123-126.
} 
exclusivamente la habilidad se predicar correctamente para discriminar los errores que estamos considerando no es suficiente. Si fuera correcto que el error del Nazi honesto Z1 pudiera ser un error de prohibición, entonces ello dejaría abierto como tendría que responder el derecho penal frente a este tipo de situaciones por la vía de la admisión de un error de prohibición. En efecto, en la medida que se afirme que a Z1 le fue inevitable creer que Z2 era inferior, Z1 podría sostener que no le fue posible formarse una intención de evitar matar a Z2. Por el contrario, si se considera que la condición (3.2) es aplicable, entonces no procede la figura del error en este caso: el derecho exige que la creencia sobre la juridicidad de la conducta sea razonable y, si no lo es, no puede haber error de prohibición.

Desde el punto de vista de la razonabilidad, el caso del nazi honesto es similar cuando el sujeto mata por creencias causadas por un desorden mental. Considérese el siguiente caso: si X4 mata a Y porque escuchó a dios que le decía "mata a Y porque Y es la reencarnación del mal y si no lo haces vendrá el apocalipsis", esto no significa que esa creencia pueda imponerse a la comunidad política haciendo inaplicable la respuesta penal por la vía del error. Incluso si es correcto que $\mathrm{Z} 1$ no cree que $\mathrm{Z} 2$ es una persona con derecho a la vida para los efectos de la aplicación de las disposiciones sobre homicidio, esto no significa que no pueda aplicarse una pena y en consecuencia imponer Z1 su comprensión del mundo a las prácticas sociales de la comunidad en la que su conducta despliega sus efectos. La creencia de Z1 tampoco es razonable (para la comunidad). Ciertamente que en estos dos casos hay diferencias. Como dijimos al comienzo, el error de prohibición y la inimputabilidad por causa de un desorden mental son situaciones diferentes y tienen respuestas diferentes por parte del derecho penal, pero no obstante sus causas sean diversas, el caso de Z1 y el de X4 son semejantes en que ni Z1 ni X4 tienen una creencia razonable de la juridicidad de su conducta. Por lo tanto, si dichas dichas creencias no son razonables, entonces no derrotan la presunción de conocimiento de la antijuridicidad del comportamiento. En consecuencia, Z1 no actúa con error de prohibición y X puede ser un candidato apto para una medida de seguridad.

Ahora bien, esta exigencia de razonabilidad no se restringe al conocimiento de la antijuridicidad de la conducta, es decir a (2.2), sino que también se aplica a (2.1). Para observar esto considérese el caso del nazi honesto Z3. El nazi honesto Z3 ha sido educado en la ideología que $Z 4$ no es una persona. Esto es, $Z 3$ no cree que $Z 4$ es una persona inferior que debe tratar a Z3 con muchísimo respeto, porque en dicho caso Z3 satisfaría (2.1) pero no (2.2) y no habrían diferencias entre Z3 y Z1. En el presente caso Z3 cree honestamente que cuando mata a Z4 no está matando a una persona ${ }^{37}$. En otras palabras, a diferencia del caso de Z1 que no satisface la condición (2.2), en este caso Z3 no satisface la condición (2.1). Recordemos la condición (2.1). Esta condición se refiere a la capacidad de $Z 3$ de creer que conducta esta realizando, en otras palabras, de la capacidad de actuar dolosamente. Sin embargo, si Z3 cree que cuando mata a Z4 no está acuchillando a una persona, entonces sus creencias son paralelas al caso en que X1, por causa de un desorden mental, cree que al matar a $Y$ no está matando a una persona sino faenando una vaca. La pregunta en estos casos es la siguiente: ¿actúan Z3 y X1 con error de tipo? No, ni X ni Z3 actúan con error de tipo. La razón es que ninguna de estas creencias es razonable. Esto sugiere que la razonabilidad aparece analíticamente de manera previa a (2.2), es decir, la razonabilidad es una exigencia epistémica aplicable no solo a las creencias que tienen por objeto una conducta caracterizada como antijurídica, sino también a las creencias que tienen por objeto una conducta determinada. Esto requiere adicionar una sub-condición a (3):

(3.1) la creencia (2.1) es una creencia razonable

\footnotetext{
${ }^{37}$ Es muy interesante notar, como se vio al comienzo de este trabajo, que para efectos de satisfacer la descripción de una conducta dolosa no se requiere que $X$ actúe, incluso cuando se trata de dolo directo, con un "estado mental ocurrente". Es decir, no se trata que X tenga un determinado "estado mental actual" cuyo objeto sea un determinado estado de hecho para satisfacer el dolo, por que el dolo no necesariamente debe ser ocurrente o actual. De lo contrario, si se requiriese que el dolo sea un "estado mental actual", entonces $Z 3$ no lo tendría porque $Z 3$ cree honestamente que $Y$ no es una persona y, por lo tanto, que no comete homicidio si mata a Y. Ahora bien, si el dolo no es un estado intencional actual, entonces es un estado disposicional y si esto es correcto, ¿significa que cuando sancionamos a $X$ por una conducta dolosa estamos considerando algo más que su estado mental?, ¿estamos considerando como X "es" y como es su carácter?
} 
Esta adición a (2.1) es lo que permite responder a la pregunta por el caso objeto de esta investigación: el inimputable que actúa con error de tipo. Este es el caso de X1 quien, al matar, cree que faena una vaca pero también es el caso de Z3. De acuerdo a la exigencia de razonabilidad, ni X1 ni Z3 actúan con error de tipo, porque para actuar con error de tipo deben satisfacer (3.1), y como sus creencias son irrazonables no actúan con error de tipo. La consecuencia que se sigue de la inexistencia del error de tipo, nuevamente, será diferente para ambos: posiblemente Z3 pueda ser considerado un candidato idóneo para una pena mientras que X1 para una medida de seguridad. Como puede notarse, la exigencia de razonabilidad ofrece una respuesta general a los casos complejos que se han considerado en este trabajo. Pero la exigencia de razonabilidad no solo ofrece una respuesta plausible a los casos problemáticos de error de tipo y error de prohibición, sino que además no presenta los problemas de la tesis asociada a Etcheberry, porque no establece una interpretación objetivista y discriminatoria inadmisible del principio de culpabilidad. Tampoco presenta las dificultades que se asociaron a Cury, porque admitir la procedencia de una medida de seguridad al caso de X1 sería consistente con nuestras prácticas de responsabilidad, de acuerdo a las cuales una persona que comete un delito causado por un desorden mental es precisamente el tipo de persona apta para ser destinataria de estas medidas, aún cuando obre con error de tipo.

No constituye objeción a la postura teórica desarrollada en esta investigación que es el mismo tipo de exigencia que se utiliza tanto para negar el error de prohibición como el error de tipo, tanto en el caso de Z3 como el de X1. La razón la ha puesto de manifiesto Mañalich, quien ha demostrado convincentemente la conexión que existe entre (2.1) y (2.2) $)^{38}$. Básicamente Mañalich sostiene que la condición (2.2) depende de que se obtenga la condición (2.1), en otras palabras, que el conocimiento de la antijuridicidad de la conducta depende que el sujeto haya conocido que tipo de comportamiento desplegaba. Es decir, si la creencia sobre la antijuridicidad de la conducta está construida sobre la creencia de la conducta típica, entonces la primera hereda la clase de creencia que es necesaria para satisfacer el dolo: la creencia razonable. Dicho de manera diferente, es precisamente porque la creencia sobre la conducta debe ser una creencia razonable (3.1) que emerge la necesidad de satisfacer la condición (3.2). En consecuencia, la razonabilidad del conocimiento de la antijuridicidad de la conducta parece derivarse de la razonabilidad del conocimiento de la conducta que se realiza.

En suma, no es correcto concluir que X1 actúa con error de tipo cuando su creencia sobre la conducta que realiza es irrazonable. Si la irrazonabilidad de la creencia se explica en que X1 tiene un desorden mental, entonces $X 1$ no es inmune a los mecanismos de responsabilidad/tratamiento penal. Por el contrario, que X1 no pueda satisfacer la condición (3.1) es precisamente lo que puede facultar al Estado, siempre que se cumplan los requisitos adicionales del art. 455 del CPP, para aplicar una medida de seguridad. En efecto, el desorden mental ha afectado la generación apropiada de creencias, y es este factor lo que vuelve a X1 en una persona socialmente incompetente y si es peligroso/a debería hacerse acreedor/a de una medida de seguridad. Una vez identificada la incapacidad clave para la culpabilidad, en este caso cognitiva y vinculada a la apropiada generación de creencias, la exigencia de razonabilidad es capaz de precisar, como de otro modo no se podría, la razón por la cual consideramos a X1 como inimputable y porque razón podría ser un candidato apto para una medida de seguridad. Pero, además, la exigencia de razonabilidad demuestra ser aplicable no solamente al caso en que X1 actúa con error de tipo cuando este es causado por un desorden mental, sino que también es aplicable cuando Z1 y Z3 no tienen un desorden mental pero cuyas creencias no son razonables, sean que no satisfagan (2.2) o (2.1). Es decir, las creencias irrazonables no solo son la provincia del inimputable, porque el imputable también puede tener creencias irrazonables.

${ }^{38}$ La tesis está en general expuesta en su trabajo MAÑALICH (2011), pp. 98-104, pero todavía sostiene esta tesis, véase MAÑALICH (2019), p. 304. 
El esquema definitivo de condiciones epistémicas que deben satisfacerse para la responsabilidad penal, y que han sido analizadas en este trabajo quedaría de la siguiente manera:

(1) P1 es verdadero

(2) $X$ cree que $P 1$ es verdadero

(2.1) $X$ cree que realiza la conducta descrita en $P 1$

(2.2) $X$ cree que realiza la conducta descrita en P1 y que P1 es antijurídica

(3) La creencia de $X$ está justificada

(3.1) la creencia (2.1) es una creencia razonable

(3.2) la creencia (2.2) es una creencia razonable

De acuerdo a este esquema hay una conducta tipificada como delito que $X$ ejecuta (1); si $X$ cree que ejecuta $P 1$ entonces su conducta es constitutiva de dolo directo (2.1); si $X$ no cree que ejecuta P1 todavía podría haber dolo (eventual) si hay evidencia suficiente para concluir que la adscripción de la creencia podría estar justificada (3); si X cree que ejecuta P1 y cree que P1 es antijurídica su conducta es dolosa y culpable (2.2); si X no satisface (2.1) por causa de un desorden mental no hay error de tipo si la creencia no es razonable (3.1); si X no satisface (2.1) no por causa de un desorden mental no hay error de tipo si la creencia no es razonable (3.1); si $X$ no satisface (2.2) no por causa de un desorden mental no hay error de prohibición si la creencia no es razonable (3.2).

\section{Conclusión}

Este trabajo ha perseguido sentar las bases para una discusión más rica y provechosa de la imputabilidad en el contexto de un desorden mental, específicamente el requerimiento que el sujeto haya conocido que su comportamiento era antijurídico. Así mismo se han examinado como diversas concepciones sobre la imputabilidad, ofrecen distintas respuestas a los casos de los posibles errores que pueden concurrir cuando el sujeto es inimputable por causa de un desorden mental y también ciertos casos especiales en que el sujeto es imputable. Si bien se trata de casos numéricamente poco habituales, la importancia de su examen es que permite revisar y evaluar las diferentes concepciones teóricas utilizadas por la doctrina chilena y las diferentes racionalizaciones que estas entregan del sistema jurídico-penal cuando responde a este tipo de hechos. Efectivamente, examinar estos casos es un escenario provechoso donde poder evaluar diferentes concepciones del derecho penal de acuerdo al tipo de respuesta que ofrecen a los problemas que se presentan. $Y$ aquí, al considerar la plausibilidad de estas respuestas ya no se trata de hecho de un ejercicio que exclusivamente interese al dogmático/a del derecho penal, sino que interesa a cualquier participante de dicha práctica. Es posiblemente en este contexto donde la exigencia de razonabilidad demuestra su rendimiento: justifica porque razón ciertos casos de error de tipo y error de prohibición deben ser rechazados y bajo que condiciones deben ser admitidos.

La exigencia de razonabilidad tiende a ajustarse a la práctica jurisprudencial en la medida que se trata de un criterio para determinar la evitabilidad o vencibilidad de ciertos errores. Sin embargo, esta tiene implicaciones más generales y sustantivas que requieren de mayor discusión. Cualquier tesis que considere las creencias que son objeto de la imputación subjetiva como de la culpabilidad en términos de actitudes no ocurrentes, admite que estas creencias sean disposicionales, y si este es el caso, entonces debe enfrentarse la ardua pero honesta tarea de ofrecer una comprensión adecuada de uno de los principios que pareciera encuentran mayor adhesión en la doctrina y la jurisprudencia: el principio del derecho penal del hecho. De acuerdo al derecho penal del hecho, la persona puede ser hecha penalmente responsable en base a elecciones que están en mayor o menor medida bajo su control, proscribiendo considerar el carácter de la persona para dicho fin. No obstante, considerar que las creencias son, al menos en parte, actitudes disposicionales y que como tales cumplen un rol crucial en el establecimiento de la condiciones de responsabilidad penal, implica re-interpretar los hechos por los cuales las 
personas son responsables como hechos que deben ser caracterizados parcialmente en términos del carácter.

Naturalmente, investigar cuales podrían ser los efectos de entender el dolo y la culpabilidad como elementos parcialmente disposicionales no puede desarrollarse en este trabajo. Sin embargo, de ser así el caso, parece resultar que nuestro derecho penal no solo sanciona la conducta intencional ocurrente, sino que también considera dolosas aquellas conductas explicadas en términos de disposiciones del agente, en términos de como cree, en términos de sus prejuicios, de sus inhabilidades e incapacidades, en suma, en términos de la propia identidad que la persona ha forjado en su vida. Esto parece conducir a comprender la práctica efectiva del derecho penal no en términos incuestionablemente adheridos al derecho penal del hecho, sino a entender al derecho penal comprometido (también) con la evaluación del carácter, como un derecho que quizás no puede (o no quiere) renunciar al derecho penal de autor.

\section{BIBLIOGRAFÍA CITADA}

AleXY, ROBERT (2007): Teoría de la Argumentación Jurídica. Teoría del Discurso Racional como Teoría de la Fundamentación Jurídica (Lima, Palestra).

Artaza Varela, Osvaldo, y Carneval Rodríguez, Raul (2018): “¿Incide la inimputabilidad en la atribución del dolo? Eventuales repercusiones en las medidas de seguridad", en: Opinión Jurídica (Vol. 17, № 34), pp. 21-43.

AUDI, ROBERT (1998): Epistemology: A Contemporary Introduction to the Theory of Knowledge (New York, Routledge).

BASCUÑÁN, ANTONIO (1998): “Observaciones sobre la Ciencia del Derecho", en: Revista de Derecho y Humanidades (Vol. 6), pp. 15-25.

Bustos, JUAN (1987): Control Social y Sistema Penal (Barcelona, PPU). (1994): Manual de Derecho Penal Parte General (Barcelona, PPU).

COHEN, JONATHAN (1992): An Essay on Belief and Acceptance (Oxford, Oxford University Press).

CousiÑo, LuIS (1975): Derecho Penal Chileno (Santiago, Editorial Jurídica de Chile), Tomo I.

Couso, JAIME (2006): Fundamentos del Derecho Penal de Culpabilidad (Valencia, Tirant Lo Blanch).

CURY, EnRIQUe (2005): Derecho Penal Parte General, 7ạ edición (Santiago, Editorial Jurídica de Chile).

DANCY, JONATHAN (1985): An Introduction to Contemporary Epistemology (Oxford, Blackwell).

DAVIDSON, DONALD (1963): “Actions, Reasons, and Causes", en: The Journal of Philosophy (Vol. 60, № 23), pp. 685-700.

ETCheBerRY, Alfredo (1997): Derecho Penal, Parte General, 3a edición (Santiago, Editorial Jurídica de Chile), tomo I.

FalCONe SALAS, Diego (2007): “Una Mirada Crítica a la Regulación de las Medidas de Seguridad en Chile", en: Revista de Derecho de la Pontificia Universidad Católica de Valparaíso (Vol. 29), pp. 235-256.

FerRAJOlI, LUIGI (2013): Principia iuris. Teoría del derecho y de la Democracia. 1. Teoría del Derecho (Madrid, Trotta).

Fernández Ruiz, José Manuel (2021A): "Los Desórdenes Mentales en el Código Penal Chileno: un Estudio Sobre la Inimputabilidad", en: Revista de Derecho (Valdivia) (pendiente de publicación). 
(2021B): "Cultural Defences, An Examination and Critique", en: Ratio luris (pendiente de publicación).

GARRIDo MontT, MARIo (2003): Derecho Penal, Parte General, 3a edición (Santiago, Editorial Jurídica de Chile), tomo II.

Hetherington, StePhen (2016): Knowledge and the Gettier Problem (Cambridge, Cambridge University Press).

Hutto, Daniel (2015): “Contentless Perceiving: The Very Idea", en: Campbell, Michael y O'Sullivan, Michael (Eds.), Wittgenstein and Perception (London, Routledge), pp. 63-83.

Kallestrup, JesPer (2020): "The Epistemology of Testimonal Trust", en: Phenomenology and Philosophical Research (Vol. 100), pp. 1-25.

Kelly, Thomas (2014): "Evidence". Disponible en: https://stanford.io/3gDrz16 [visitado el 7 de agosto de 2020].

KINDHAÜSER, URS (1999): “Acerca de la Distinción entre Error de Hecho y Error de Derecho", en: VV.AA., El Error en el Derecho Penal (Buenos Aires, Ad-Hoc), pp. 139-163.

(2019): "El error sobre las circunstancias del hecho frente al error de prohibición", en: Revista de Estudios de la Justicia (Vol. 31), pp. 127-145.

KüNSEMÜlLeR, CARLOS (2001): Culpabilidad y Pena (Santiago, Editorial Jurídica de Chile).

Labatut, Gustavo (1989): Derecho Penal, 9ạ edición (Santiago, Editorial Jurídica de Chile), tomo I.

LYCAN, WILLIAM (2019): Philosophy of Language: A Contemporary Introduction, $3^{\text {rd }}$ edition (New York\&London, Routledge).

MAÑAlich RAFFo, JUAN PABLo (2011): “El delito como injusto culpable. Sobre la conexión funcional entre el dolo y la consciencia de la antijuridicidad en el derecho penal chileno", en: Revista de Derecho (Vol. 24, № 1), pp. 87-115.

(2019): “Tentativa, error y dolo. Una reformulación normológica de la distinción entre tentativa y delito putativo", en: Política Criminal (Vol. 14, № 27), pp. 296-375.

Molina FernándeZ, Fernando (2008): "Error de tipo derivado de anomalías o alteraciones psíquicas: un difícil desafío para la teoría del delito", en: Icade. Revista Cuatrimestral de las Facultades de Derecho y Ciencias Económicas y Empresariales (Vol. 74), pp. 113-144.

NAGel, JenNifer (2013): "Knowledge as a Mental State", en: Gendler, Tamar y Hawthorne, John (Eds.), Oxford Studies in Epistemology (Oxford, Oxford University Press), volume 4, pp. 273-308.

NÁQUIRA, JAIME (1998): Derecho Penal Parte General (Santiago, McGraw-Hill), tomo I.

NovoA, Eduardo (2005): Curso de Derecho Penal Chileno. Parte General (Santiago, Editorial Jurídica de Chile), tomo I.

Quiroga, Luis Ortiz y ArÉvalo, JaVier (2014): Las Consecuencia Jurídicas del Delito (Santiago, Editorial Jurídica de Chile).

Politoff, Sergio; Matus, Jean Pierre y Ramírez, María Cecilia (2003): Lecciones de Derecho Penal Chileno (Santiago, Editorial Jurídica de Chile).

(1997): Derecho Penal (Santiago, Editorial Conosur), tomo I.

PRITCHARD, DUNCAN (2016): Epistemology (Basingstoke, Palgrave Macmillan).

QUINE, WILlaRD (1968): “Epistemology Naturalized”, en: Quine, Willard, Ontological Relativity and Other Essays (New York, Columbia University Press), pp. 69-90. 
QuinN, WARREN (1993): Morality and Action (Cambridge, Cambridge University Press).

RAgués I VAlLès, RAMón (2004): “Consideraciones Sobre la Prueba del Dolo", en: Revista de Estudios de la Justicia (№ 4), pp. 13-26.

RoxIN, Claus (1997): Derecho Penal Parte General (Madrid, Civitas), tomo I.

SCHAPIRO, TAMAR (2014): "What are theories of desire theories of?", en: Analytic Philosophy (Vol. 55, № 2), pp. 131-150.

SCHROEDER, TIMMOTHY (2015): “Desire". Disponible en https://stanford.io/3tXdUpk [visitado el 7 de agosto de 2020].

SEARLE, JoHn (2004): La Construcción de la Realidad Social (Barcelona, Paidos).

Silva SÁnCHeZ, Jesús MARía (2003): “¿Medidas de seguridad sin imputación subjetiva? Consecuencias de la incidencia de anomalías y alteraciones psíquicas en niveles sistemáticos distintos de la imputabilidad", en: Silva Sánchez, Jesús, Normas y Acciones en Derecho Penal (Buenos Aires, Hammurabi), pp. 109-133.

SMITH, Holly (2011): "The Moral Clout of Reasonable Beliefs", en: Timmons, Mark (Ed.), Oxford Studies in Normative Ethics (Oxford, Oxford University Press), pp. 1-25.

SOTOMAYOR, JUAN OBERTO (2016): “Fundamento del dolo y ley penal: una aproximación crítica a las concepciones cognitivo/normativas del dolo, a propósito del caso colombiano", en: Política Criminal (Vol. 11, № 22), pp. 675-703.

SteUP, MATTHIAS (1996): Introduction to Contemporary Epistemology (New Jersey, Prentice-Hall).

Strawson, Peter (2008): "Freedom and Resentment", en: Strawson, Peter, Freedom and Resentment and Other Essays (London, Routledge), pp. 1-28.

TAPIA, PATRICIA (2013): "Las medidas de seguridad. Pasado, presente y ¿futuro? de su regulación en la legislación chilena y española”, en: Política Criminal (Vol. 8, № 16), pp. 574-599.

Van Weezel, Alex (2008): Error y Mero Desconocimiento en Derecho Penal (Santiago, Editorial Jurídica de Chile).

Vargas, Tatiana (2010): Manual de Derecho Penal Práctico. Teoría del Delito con Casos (Santiago, LegalPublishing).

(2016). "Breves Reflexiones del Error Desde la Jurisprudencia", en: Cárdenas, Claudia y Ferdman, Jorge (Coords.), El Derecho Penal Como Teoría y Como Práctica. Libro En Homenaje a Alfredo Etcheberry Orthusteguy (Santiago, LegalPublishing), pp. 323-351.

Velleman, DaVid (1992): “The Guise of the Good”, en: Nous (Vol. 26, № 1), pp. 3-26.

WILAND, ERIC (2010): Reasons (London/New York, Continuum).

Williams, Michael (2001): The Problem of Knowledge: A Critical Introduction to Epistemology (Oxford, Oxford University Press). 